\title{
Microstructure and Magnetic Properties of Iron Oxide Nanoparticles Prepared by Wet Chemical Method
}

\author{
D. Satula ${ }^{a}$, B. KAlska-SzOstKo ${ }^{b}$, K. Szymański ${ }^{a}$, \\ L. DoBrzyŃSKI ${ }^{a, c}$ AND J. KOZUBOWsKI ${ }^{d}$ \\ ${ }^{a}$ Faculty of Physics, University of Białystok \\ Lipowa 41, 15-424 Białystok, Poland \\ ${ }^{b}$ Institute of Chemistry, University of Białystok \\ Hurtowa 1, 15-399 Białystok, Poland
}

${ }^{c}$ The Soltan Institute of Nuclear Studies, 05-400 Otwock-Świerk, Poland

${ }^{d}$ Warsaw University of Technology

Faculty of Materials Science and Engineering

Wołoska 141, 02-507 Warszawa, Poland

The magnetic properties of iron oxide nanoparticles prepared by the chemical decomposition of the iron chlorides with the ratio $\mathrm{Fe}^{3+} / \mathrm{Fe}^{2+}=2.25$ were studied by means of transmission electron microscopy, X-ray diffraction and Mössbauer spectroscopy in- and without external magnetic field. The transmission electron microscopy studies show that the nanoparticles have spherical shape with diameter about $13 \mathrm{~nm}$. The transmission electron microscopy and X-ray diffraction patterns are composed of lines that could be indexed within the cubic spinel structure. The room temperature Mössbauer spectrum shows the coexistence of the broad magnetically split part and superparamagnetic doublet. The in-field Mössbauer measurements carried out in the temperature range from room temperature down to $13 \mathrm{~K}$ show a gradual increase in the spin canting on the surface of the nanoparticles and decrease in the intensity of the superparamagnetic doublet. The sample was subjected to annealing process performed at elevated temperature in air atmosphere in order to change the microstructure of the nanoparticles and in consequence to change the magnetic properties of the sample. The annealing process leads to the decrease in the value of quadrupole splitting of the superparamagnetic doublets.

PACS numbers: 76.80.+y, 61.46.Df, 75.75.+a

\section{Introduction}

The magnetic nanoparticles are of great interest because of their novel properties, which differ considerably from that observed ones in the bulk materials [1]. 
The few nanometer size causes that the surface effects strongly influence magnetic properties of the nanoparticles. The fundamental mechanisms that govern their behavior are connected with single particle properties and collective interparticles interactions. It was reported that the low symmetry of the surface atoms and possible distribution of interior defects and/or microstructure can lead to the spin canting $[2,3]$. Moreover, the interparticle interaction can lead to suppression of the superparamagnetic relaxation and may result in spin-glass-like ordered state at low temperatures $[4,5]$. The interparticle interactions have been proposed to stabilize the magnetic order both at the nanoparticle's core and surface [6].

The aim of this work was to investigate the behavior of magnetic moments of iron atoms with decrease in temperature and an impact of the annealing process on the microstructure and in consequence on the hyperfine parameters of the iron oxide nanoparticles. The thermal annealing in air can lead to the transformation from magnetite - maghemite to hematite microstructure of the outer parts of iron oxide nanoparticles which should lead to a change of the magnetic properties - stabilization of the magnetic order of the interior nanoparticles in particular. It is expected that the gradual annealing process should influence the surface first and then the core of the nanoparticles.

\section{Experiment}

The iron oxide nanoparticles were synthesized following main steps from Massart's methods [7]. The particle production is divided into two main procedures. Initially the chlorides are hydrolyzed to certain hydroxides. The reaction takes place in two separated flasks. In the second step, the condensation occurs where the reagents are mixed in one bottle at elevated temperature up to 85$90^{\circ} \mathrm{C}$. In the meantime the surfactant such as tetrabutyloammonium hydroxide (TBAOH) is added to the solution. The nanoparticles extracted from solution were characterized by low- and high-resolution electron diffraction (TEM), X-ray diffraction (XRD) and Mössbauer spectroscopy in- and without external magnetic field. Transmission electron micrographs were taken out on a JEM 3010 microscope. The Mössbauer spectra were obtained using the equipment with a conventional constant acceleration mode spectrometer with a ${ }^{57} \mathrm{Co}$ source in chromium matrix. The reference spectra were obtained on $\alpha$-Fe foil at room temperature. In-field measurements were taken with the use of external magnetic field of $1.3 \mathrm{~T}$ and $1 \mathrm{~T}$ parallel to the $\gamma$-ray direction. Low temperature measurements were carried out with the use of closed cycle refrigerator equipped with antivibrational shroud.

The powder sample was mixed with boron nitride and epoxy glue to obtain homogeneous tablets. The specimens were annealed in air atmosphere at temperature $423 \mathrm{~K}$ for $2 \mathrm{~h}$. The procedure was repeated twice. 


\section{Results and discussion}

The TEM images of the as prepared sample are shown in Fig. 1. The particles are roughly spherical and agglomerated. The distribution of the diameters is presented in Fig. 2. The estimated average size of the particles is $(12.8 \pm 1.5) \mathrm{nm}$. The high resolution picture presents clearly that the particles' cores are single crystalline. The shell of the particles is not separated. The electron diffraction patterns shows (220), (311), (400), (422), (511) and (440) rings originating from the magnetite $\left(\mathrm{Fe}_{3} \mathrm{O}_{4}\right)$ and/or maghemite $\left(\gamma-\mathrm{Fe}_{2} \mathrm{O}_{3}\right)$ structures since the positions of the diffraction patterns are almost the same in both of them [8]. The obtained ring pattern does not show any additional reflexes (rings) which could be associated with $\alpha-\mathrm{Fe}_{2} \mathrm{O}_{3}$ or other oxides. Small amount of crystalline organic ingredients connected with surfactant is also noticed. These results were confirmed by X-ray experiment.

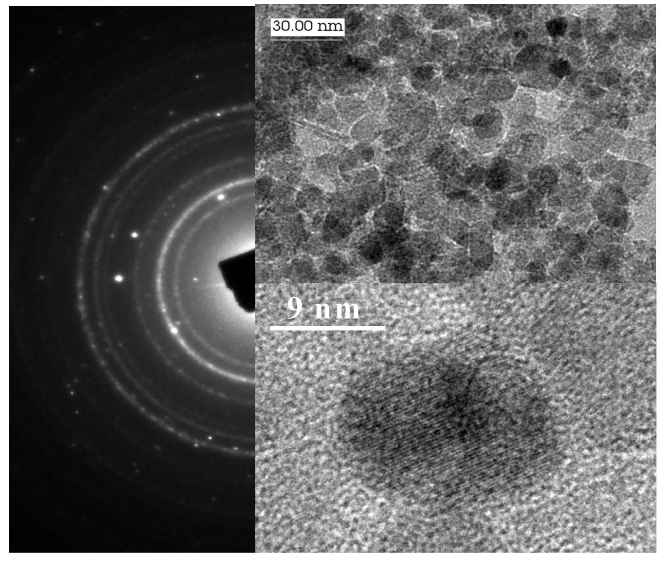

Fig. 1. TEM images and electron diffraction pattern of the sample.

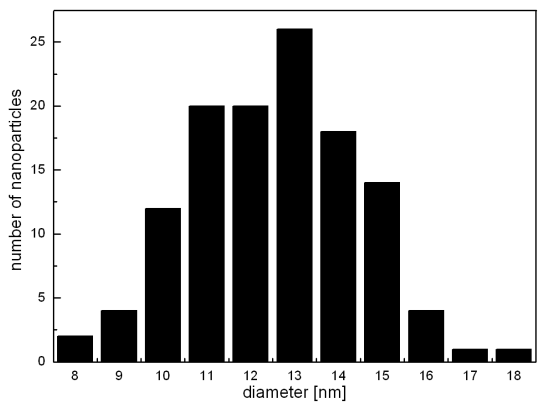

Fig. 2. Particles size distribution obtained from TEM images. 
The Mössbauer spectra measured in- and without external field at room temperature are presented in Fig. 3. As one can see, the spectrum measured without external magnetic field consists of broad magnetic part and a doublet. The presence of the doublet which could be due to some superparamagnetic relaxation is rather surprising. For such materials like magnetite and maghemite which crystallize in regular structure, the superparamagnetic relaxation should be rather seen as a single line than doublets - the situation known from the measurements on bulk magnetite above $T_{\mathrm{c}}[9]$. The superparamagnetic relaxation often observed in nanoparticles is related to the particles' size. Alternatively, one can explain the presence of superparamagnetic doublet as showing that remarkable part of $\mathrm{Fe}$ atoms is located in the region of the nanoparticle surface and because of broken cubic symmetry these atoms are exposed to nonzero electric field gradient.

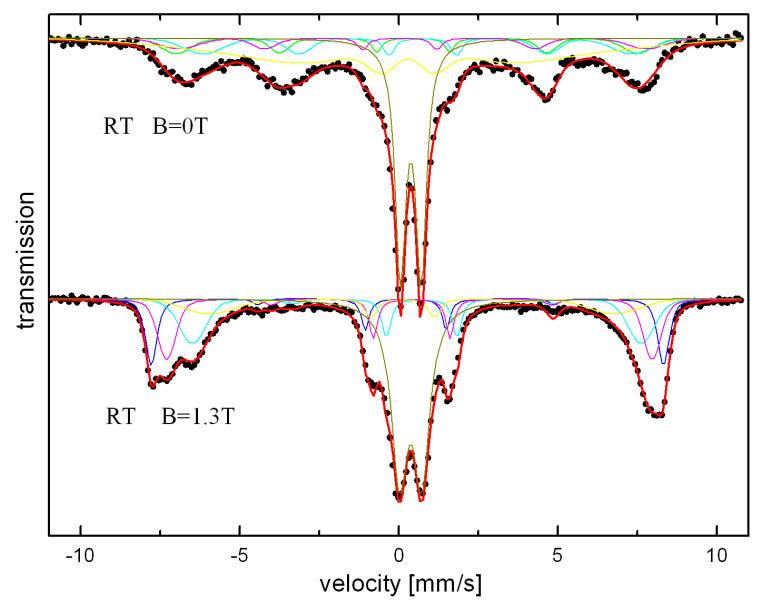

Fig. 3. The Mössbauer spectra measured at room temperature (RT) in- and without external magnetic field parallel to gamma beam.

The in-field measurements show that the magnetic part of spectra is well resolved and individual components can be extracted. Moreover, the lines 2 and 5 disappear which means that the hyperfine fields are oriented along gamma beam direction. The spectra were fitted using four sextets and one doublet. These two sextets come from the iron atoms at $\mathrm{A}$ and $\mathrm{B}$ positions connected with two different iron positions in magnetite, one sextet which comes from iron in maghemite and one broad sextet connected with superparamagnetic relaxation processes. The relative intensity of the doublet is $(37 \pm 4) \%$ of the spectra and within the experimental accuracy does not change for in- and without external magnetic field measurements. Assuming that the outer layer is solely responsible for the presence of the doublets, one can estimate that the thickness of this layer must be $(2.0 \pm 0.5) \mathrm{nm}$. This value is over twice larger than the lattice parameter for maghemite which for nanoparticles varies from 0.833 to $0.838 \mathrm{~nm}$ [10]. 
The Mössbauer spectra measured in the temperature range $300 \mathrm{~K}$ down to $13 \mathrm{~K}$ are presented in Fig. 4. Two main features follow from inspection of the observed spectral shapes. The quadrupole components decrease and disappear at $T=50 \mathrm{~K}$ and the gradual increase in the intensities connected with the lines 2 and 5 is observed when the temperature decreases. This can be interpreted as suppression of the superparamagnetic relaxation processes and formation of the static disordered spin structure. This confirms that the doublet is connected with the atoms located at the outer part of the particle. The gradual increase in the intensities of the lines 2 and 5 with decrease in the temperature were observed in maghemite nanoparticles $(7 \mathrm{~nm})$ in the work [11] and interpreted as strongly canted iron magnetic moments from B-sites ions.

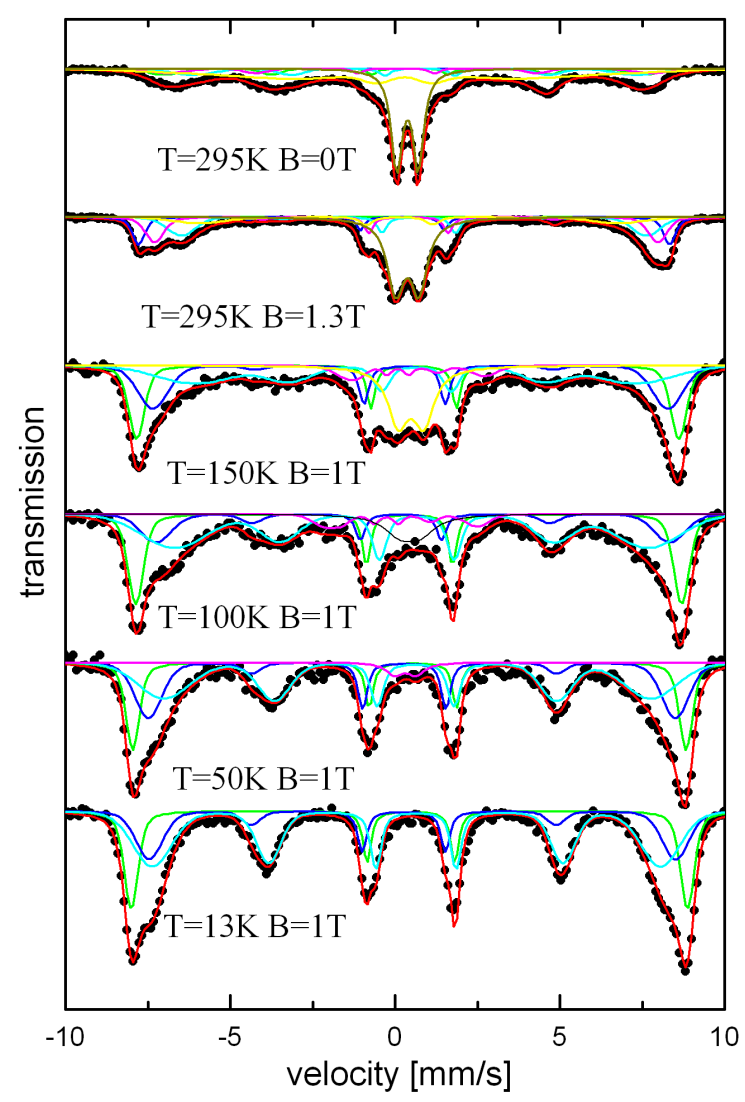

Fig. 4. The Mössbauer spectra measured at temperature range $13 \mathrm{~K}$ to RT.

The Mössbauer spectra measured at room temperature for sample subjected to different annealing process are presented in Fig. 5. The intensities of the superparamagnetic doublets decrease with increase in the annealing time. Moreover, the results show that value of the quadrupole splitting decreases from 


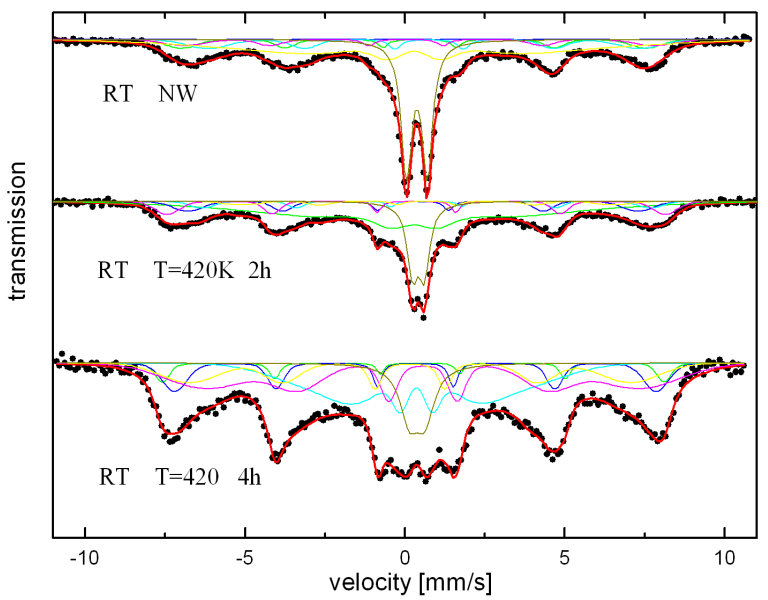

Fig. 5. The room temperature Mössbauer spectra measured for samples subjected to different annealing processes.

$(0.65 \pm 0.03) \mathrm{mm} / \mathrm{s}$ for as prepared sample to $(0.37 \pm 0.04) \mathrm{mm} / \mathrm{s}$ for sample annealed for $2 \mathrm{~h}$. Further annealing does not change the value. The decrease in quadrupole splitting can be caused by a change of the outer layer morphology or by coating the particles by hematite. The small components of the hematite were detected in the in-field Mössbauer spectra of the sample subjected to $4 \mathrm{~h}$ annealing. We cannot exclude a possibility of surfactant decomposition resulting in an increase in dipolar interaction between the particles.

\section{Conclusions}

TEM measurements, X-ray diffraction and Mössbauer spectroscopy show that using the chemical wet method the magnetite/maghemite iron oxide nanoparticles with the size of $(12.8 \pm 1.5)$ nm were produced. The Mössbauer spectra at room temperature consist of the superparamagnetic doublet and the magnetic part. The applied external magnetic field exerts strong influence on the magnetic part of the spectra and does not change the superparamagnetic part. The Mössbauer measurements carried out in the temperature from room temperature down to $13 \mathrm{~K}$ show gradual suppression of the superparamagnetic component and formation of static spin disordered structure. The procedure of annealing of the nanoparticles in air leads to the change of the value of the quadrupole interaction of the iron atoms in the outer layer of the nanoparticles and partially suppression of the superparamagnetic phenomenon.

\section{Acknowledgments}

The work was partially supported as a research project by the funds allocated for scientific research for years 2008-2011. 


\section{References}

[1] J.L. Dormann, D. Fiorani, E. Tronc, Adv. Chem. Phys. 98, 283 (1997).

[2] J.M.D. Coey, Phys. Rev. Lett. 27, 1140 (1971).

[3] S. Morup, J. Magn. Magn. Mater. 266, 110 (2003).

[4] S. Mørup, F. Bodker, P.V. Hendriksen, S. Linderoth, Phys. Rev. B 52, 287 (1995).

[5] Y. Sun, M.B. Salamon, K. Garnier, R.S. Averback, Phys. Rev. Lett. 91, 167206 (2003).

[6] V. Skumryev, S. Stoyanov, Y. Zhang, G. Hadjipanayis, D. Givord, J. Nauues, Nature 423, 850 (2003).

[7] R. Massart, V. Cabuil, J. Chim. Phys. 84, 967 (1987).

[8] S. Sun, H. Zeng, D.B. Robinson, S. Raoux, P.M. Rice, S.X. Wang, G. Li, J. Am. Chem. Soc. 126, 273 (2004).

[9] J.M. Florez, J. Mazo-Zuluaga, J. Restrepo, Hyperfine Interact. 161, 161 (2005).

[10] S. Nasrazadani, A. Raman, Corros. Sci. 34, 1355 (1993).

[11] Ö. Helgason, H. Rasmusen, S. Mørup, J. Magn. Magn. Mater. 302, 413 (2006). 